\title{
Optimal Error Estimates of the Crank-Nicolson Scheme for Solving a Kind of Decoupled FBSDEs
}

\author{
Zhe Wang, Yang Li \\ College of Science, University of Shanghai for Science and Technology, Shanghai, China \\ Email:wdzhao@sdu.edu.cn
}

How to cite this paper: Wang, $\mathrm{Z}$. and $\mathrm{Li}$, Y. (2018) Optimal Error Estimates of the Crank-Nicolson Scheme for Solving a Kind of Decoupled FBSDEs. Journal of Applied Mathematics and Physics, 6, 338-346. https://doi.org/10.4236/jamp.2018.62032

Received: November 22, 2017

Accepted: February 5, 2018

Published: February 8, 2018

Copyright $\odot 2018$ by authors and Scientific Research Publishing Inc. This work is licensed under the Creative Commons Attribution International License (CC BY 4.0).

http://creativecommons.org/licenses/by/4.0/ (c) (i) Open Access

\begin{abstract}
In this paper, under weak conditions, we theoretically prove the second-order convergence rate of the Crank-Nicolson scheme for solving a kind of decoupled forward-backward stochastic differential equations.
\end{abstract}

\section{Keywords}

Forward Backward Stochastic Differential Equations, Second-Order Scheme, Error Estimate, Trapezoidal Rule

\section{Introduction}

The existence and uniqueness of the solution for nonlinear backward stochastic differential equations (BSDEs) were first proved by [1]. Since then, BSDEs have been extensively studied by many researchers. At the same time, many applications have been found. In [2], Peng obtained the relation between the backward stochastic differential equation and the parabolic partial differential equation (PDE). By using the relation between the BSDE and PDE, a four step scheme was proposed in [3]. In [4], some simple numerical schemes were proposed for BSDEs and half-order convergence error estimates were proved. In [5], Zhao et al. proposed some new kind of high accurate numerical method for BSDEs, which the scheme with second order convergence rate was first proposed and analyzed in [6] [7] and [8]. However, In [6] [7] [8], the authors only proved the schemes were of high order convergence for solving $y$ and $z$ with the generator $f$ not depending on $z$. In [8], the authors proved the errors measured in the $L^{p}(p \geq 1)$ sense are of high order convergence in solving $y$ and $z$. In [9], the authors rigorously obtained the error estimate of Crank-Nicolson scheme for solving generalized BSDEs, and theoretically proved the high convergence rates for solving $y$ and $z$. In this paper we consider the following decoupled FBSDE 


$$
\left\{\begin{array}{l}
X_{r}^{t, x}=x+\int_{t}^{r} b\left(s, X_{s}^{t, x}\right) \mathrm{d} s+\int_{t}^{r} \sigma\left(s, X_{s}^{t, x}\right) \mathrm{d} \tilde{N}_{s}, \quad(\mathrm{SDE}) \\
Y_{r}^{t, x}=\varphi\left(X_{T}^{t, x}\right)+\int_{r}^{T} f_{s}^{t, x} \mathrm{~d} s-\int_{r}^{T} \int_{\mathcal{E}} U_{s}^{t, x} \mathrm{~d} \tilde{N}_{s}, \quad \text { (BSDE) }
\end{array}\right.
$$

where the generator $f_{s}^{t, x}=f_{1}\left(s, X_{s}^{t, x}, Y_{s}^{t, x}\right)+h(s) \Gamma_{s}^{t, x}$ with $\Gamma_{s}^{t, x}=\int_{\mathcal{E}} U_{s}^{t, x} \rho(e) \lambda(\mathrm{de})$ and $h:[0, T] \rightarrow R^{d}$.

In this paper, we will consider the BSDEs (1.1). Under weaker conditions, we proved the Crank-Nicolson scheme has second-order convergence rate for solving the decoupled FBSDEs. In Section 2, we introduce some preliminaries and notation, and introduce the scheme in Section 3. In Section 4, we prove that the scheme is of second-order convergence in solving $y$ and of first-order convergence in solving $\Gamma$ for the FBSDEs (1.1).

\section{Preliminaries and Notation}

Let $T$ be a fixed positive number and $\left\{\Omega, \mathcal{F}, \mathbb{P},\left\{\mathcal{F}_{t}\right\}_{0 \leq t \leq T}\right\}$ be a complete, filtered probability space on which a standard Brownian motion $W_{t}$ is defined. Note that $\left\{\mathcal{F}_{t}\right\}_{0 \leq t \leq T}$ is the natural filtration of the Brownian motion $W_{t}$ and all the P-null sets are augmented to each $\sigma$-field $\mathcal{F}_{t}$. We denote $|\cdot|$ and $L^{2}=L_{\mathcal{F}}^{2}\left(0, T ; \mathbb{R}^{d}\right)$ as the standard Euclidean norm in $\mathbb{R}^{m}$ (or $\mathbb{R}^{m \times d}$ ) and the set of all $\mathcal{F}_{t}$-adapted and $L^{2}$-integrable processes valued in $\mathbb{R}^{d}$, respectively.

A process $\left(X_{t}, Y_{t}, \Gamma_{t}\right):[0, T] \times \Omega \rightarrow \mathbb{R}^{m} \times \mathbb{R}^{m \times d}$ is called an $L^{2}$-adapted solution of the BSDE (1.1) if it is $\left\{\mathcal{F}_{t}\right\}$-adapted and $L^{2}$-integrable, and satisfies (1.1). Now we introduce the following notations.

- $C_{b}^{l, k}$ : the set of continuously differential functions $\phi \in[0, T] \rightarrow R$ with the partial derivatives $\partial_{t}^{l_{1}} \phi$ uniformly bounded for $l_{1} \leq l$.

- $C_{P}^{l}$ : the set of continuously differential functions $\phi \in R^{d} \rightarrow R$ with all of its partial derivatives $\partial_{x}^{l_{1}} \phi$ of order up to and including $l$ have the polynomial growth.

- $\mathcal{F}_{s}^{t, x}(t \leq s \leq T): \sigma$-field generated by the Brownian motion $\left\{x+W_{r}-W_{t}, t \leq r \leq s\right\}$ starting from the time-space point $(t, x)$. When $s=t$, we use $\mathcal{F}^{t, x}$ to denote $\mathcal{F}_{T}^{t, x} \cdot \mathbb{E}[X]$ : the mathematical expectation of the random variable $X . \mathbb{E}_{s}^{t, x}[X]$ : the conditional mathematical expectation of the random variable $X$ under the $\sigma$-field $\mathcal{F}_{s}^{t, x}$, i.e., $\mathbb{E}_{s}^{t, x}[X]=\mathbb{E}\left[X \mid \mathcal{F}_{s}^{t, x}\right]$. When $s=t$, we use $\mathbb{E}_{t}^{x}[X]$ to denote $\mathbb{E}\left[X \mid \mathcal{F}_{t}^{t, x}\right]$.

- Throughout this paper, $C$ is a generic positive constant depending only on $c_{0}, T$, and upper bounds of functions $h, \varphi, f_{1}$ and their derivatives, moreover $C$ can be different from line to line.

\section{Schemes for BSDE (1.1)}

We will give a brief review on the schemes proposed in [10] for solving the BSDE (1.1).

For the time interval $[0, T]$, we introduce the following time partition: $0=t_{0}<\cdots<t_{N}=T$, and let $\Delta t=t_{n+1}-t_{n}$ and $h=\max _{0 \leq n \leq N-1} \Delta t$. From (1.1), it is 
easy to obtain that for $0 \leq n \leq N-1$,

$$
Y_{t_{n}}^{t_{n}, X^{n}}=Y_{t_{n+1}}^{t_{n}, X^{n}}+\int_{t_{n}}^{t_{n+1}} f_{s}^{t_{n}, X^{n}} \mathrm{~d} s-\int_{t_{n}}^{t_{n+1}} \int_{\mathcal{E}} U_{s}^{t_{n}, X^{n}} \mathrm{~d} \tilde{N}_{s} .
$$

where $f_{s}^{t_{n}, X^{n}}=f_{1}\left(s, X_{s}^{t_{n}, X^{n}}, Y_{s}^{t_{n}, X^{n}}\right)+g(s) \Gamma_{s}^{t_{n}, X^{n}}$. Taking the conditional mathematical expectation $\mathbb{E}_{t_{n}}^{X^{n}}[\cdot]$ on both sides of (3.1), we get

$$
Y_{t_{n}}^{t_{n}, X^{n}}=\mathbb{E}_{t_{n}}^{X^{n}}\left[Y_{t_{n+1}}^{t_{n}, X^{n}}\right]+\int_{t_{n}}^{t_{n+1}} \mathbb{E}_{t_{n}}^{X^{n}}\left[f_{s}^{t_{n}, X^{n}}\right] \mathrm{d} s .
$$

The integrand $\mathbb{E}_{t_{n}}^{X^{n}}\left[f_{s}^{t_{n}, X^{n}}\right]$ on the right-hand side of (3.2) is a deterministic smooth function of time $s$. We may use some numerical integration methods to accurately approximate the integral in (3.2). In particular, we use the trapezoidal rule to approximate the integral on the right hand side of (3.2) and obtain

$$
Y_{t_{n}}^{t_{n}, X^{n}}=\mathbb{E}_{t_{n}}^{X^{n}}\left[Y_{t_{n+1}}^{t_{n+1}, X^{n+1}}\right]+\frac{1}{2} \Delta t_{n}\left(f_{t_{n}}^{t_{n}, X^{n}}+\mathbb{E}_{t_{n}}^{X^{n}}\left[f_{t_{n+1}}^{t_{n+1}, X^{n+1}}\right]\right)+\sum_{j=1}^{2} R_{y_{j}}^{n},
$$

where

$$
\begin{aligned}
& R_{y_{1}}^{n}=\int_{t_{n}}^{t_{n+1}}\left\{\mathbb{E}_{t_{n}}^{X^{n}}\left[f_{s}^{t_{n}, X^{n}}\right]-\frac{1}{2} \mathbb{E}_{t_{n}}^{X^{n}}\left[f_{t_{n+1}}^{t_{n}, X^{n}}\right]-\frac{1}{2} f_{t_{n}}^{t_{n}, X^{n}}\right\} \mathrm{d} s, \\
& R_{y_{2}}^{n}=\mathbb{E}_{t_{n}}^{X^{n}}\left[Y_{t_{n+1}}^{t_{n}, X^{n}}-Y_{t_{n+1}}^{t_{n+1}, X^{n+1}}\right]+\frac{1}{2} \Delta t_{n} \mathbb{E}_{t_{n}}^{X^{n}}\left[f_{t_{n+1}, X^{n}}^{t_{n}}-f_{t_{n+1}}^{t_{n+1}, X^{n+1}}\right] .
\end{aligned}
$$

Let $\Delta N_{\rho, n}=\int_{\mathcal{E}} \rho(e) \tilde{N}\left(\mathrm{~d} e,\left[t_{n}, t_{n+1}\right]\right)$ for $t_{n} \leq s \leq t_{n+1}$. Then $\Delta N_{\rho, n}$ is a compensated Poisson process with mean zero and variance $s-t_{n}$. Now multiply (3.1) by $\Delta N_{\rho, n}^{\mathrm{T}}$, and take the conditional mathematical expectation $\mathbb{E}_{t_{n}}^{X^{n}}[\cdot]$ on both sides of the derived equation, we obtain by the Itô isometry formula

$$
-\mathbb{E}_{t_{n}}^{X^{n}}\left[Y_{t_{n+1}}^{t_{n}, X^{n}} \Delta N_{\rho, n}^{\mathrm{T}}\right]=\int_{t_{n}}^{t_{n+1}} \mathbb{E}_{t_{n}}^{X^{n}}\left[f_{s}^{t_{n}, X^{n}} \Delta N_{\rho, n}^{\mathrm{T}}-\Gamma_{s}^{t_{n}, X^{n}}\right] \mathrm{d} s .
$$

Based on (3.5), we have

$$
\begin{aligned}
\frac{1}{2} \Delta_{n} \Gamma_{t_{n}}^{t_{n}, X^{n}}= & -\frac{1}{2} \Delta_{n} \mathbb{E}_{t_{n}}^{X^{n}}\left[\Gamma_{t_{n+1}}^{t_{n+1}, X^{n+1}}\right]+\mathbb{E}_{t_{n}}^{X^{n}}\left[Y_{t_{n+1}}^{t_{n+1}, X^{n+1}} \Delta \tilde{N}_{\rho, n}^{\mathrm{T}}\right] \\
& +\frac{1}{2} \Delta_{n} \mathbb{E}_{t_{n}}^{X^{n}}\left[f_{t_{n+1}}^{t_{n+1}, X^{n+1}} \Delta \tilde{N}_{\rho, n}^{\mathrm{T}}\right]+\sum_{j=1}^{2} R_{\Gamma j}^{n},
\end{aligned}
$$

where

$$
\begin{aligned}
R_{\Gamma 1}^{n}= & \int_{t_{n}}^{t_{n+1}} \mathbb{E}_{t_{n}}^{X^{n}}\left[f_{s}^{t_{n}, X^{n}} \Delta \tilde{N}_{\rho, n}^{\mathrm{T}}\right] \mathrm{d} s-\frac{1}{2} \Delta_{n} \mathbb{E}_{t_{n}}^{X^{n}}\left[f_{t_{n+1}}^{t_{n}, X^{n}} \Delta \tilde{N}_{\rho, n}^{\mathrm{T}}\right] \\
& -\int_{t_{n}}^{t_{n+1}}\left\{\mathbb{E}_{t_{n}}^{X^{n}}\left[\Gamma_{s}^{t_{n}, X^{n}}\right]-\frac{1}{2} \mathbb{E}_{t_{n}}^{X^{n}}\left[\Gamma_{t_{n+1}}^{t_{n}, X^{n}}\right]-\frac{1}{2} \Gamma_{t_{n}}^{t_{n}, X^{n}}\right\} \mathrm{d} s, \\
R_{\Gamma 2}^{n}= & -\frac{1}{2} \Delta_{n} \mathbb{E}_{t_{n}}^{X^{n}}\left[\Gamma_{t_{n+1}}^{t_{n}, X^{n}}-\Gamma_{t_{n+1}}^{t_{n+1}, X^{n+1}}\right]+\mathbb{E}_{t_{n}}^{X^{n}}\left[\left(Y_{t_{n+1}}^{t_{n}, X^{n}}-Y_{t_{n+1}}^{t_{n+1}, X^{n+1}}\right) \Delta \tilde{N}_{\rho, n}^{\mathrm{T}}\right] \\
& +\frac{1}{2} \Delta_{n} \mathbb{E}_{t_{n}}^{X^{n}}\left[\left(f_{t_{n+1}}^{t_{n}, X^{n}}-f_{t_{n+1}}^{t_{n+1}, X^{n+1}}\right) \Delta \tilde{N}_{\rho, n}^{\mathrm{T}}\right] .
\end{aligned}
$$

Based on reference Equations (3.3) and (3.6), for solving the BSDEs (1.1) we introduce the following scheme.

Scheme 1 Given a random variable $Y^{N}$, solve random variables $Y^{n}$ and $Z^{n} \quad(n=N-1, N-2, \cdots, 1,0)$ backwardly by

$$
X^{n+1}=X^{n}+\sum_{\alpha \in \Gamma_{2} \backslash\{v\}} g_{\alpha}\left(t_{n}, X^{n}\right) I_{\alpha, n} ;
$$




$$
\begin{gathered}
Y^{n}=\mathbb{E}_{t_{n}}^{X^{n}}\left[Y^{n+1}\right]+\frac{1}{2} \Delta t_{n}\left(f^{n}+f^{n+1}\right) \\
\frac{1}{2} \Delta t_{n} \Gamma^{n}=-\frac{1}{2} \Delta t_{n} \mathbb{E}_{t_{n}}^{X^{n}}\left[\Gamma^{n+1}\right]+\mathbb{E}_{t_{n}}^{X^{n}}\left[Y^{n+1} \Delta \tilde{N}_{\rho, n}^{\mathrm{T}}\right]+\frac{1}{2} \Delta t_{n} \mathbb{E}_{t_{n}}^{X^{n}}\left[f^{n+1} \Delta \tilde{N}_{\rho, n}^{\mathrm{T}}\right]
\end{gathered}
$$

where $f^{n}=f_{1}\left(t_{n}, X^{n}, Y^{n}\right)+h\left(t_{n}\right) \Gamma^{n}$ for $n=N-1, N-2, \cdots, 0$.

\section{Error Estimates}

In this section, we will estimate the errors $e_{Y}^{n}=Y_{t_{n}}^{t_{n}, X^{n}}-Y^{n}$ and $e_{\Gamma}^{n}=\Gamma_{t_{n}}^{t_{n}, X^{n}}-\Gamma^{n}$ in $L^{2}$ norm, where $\left(Y_{t_{n}}^{t_{n}, X^{n}}, Z_{t_{n}}^{t_{n}, X^{n}}\right)$ is the solution of the BSDE (1.1) and $\left(Y^{n}, Z^{n}\right)$ is the solution of Scheme 1. For the sake of simplicity, we only consider one-dimensional BSDEs (i.e., $m=d=1$ ). However, all error estimates we obtain in the sequel also hold for general multidimensional BSDEs. Let $\Delta t=\max _{0 \leq n \leq N-1} \Delta t$. In our error analysis, we will use the constraint on the time partition step $\Delta t$ :

$$
\frac{\max _{0 \leq n \leq N-1} \Delta t}{\min _{0 \leq n \leq N-1} \Delta t} \leq c_{0}
$$

Let us first introduce the following lemma. Its proof can be found in the reference.

Lemma 1 Let $R_{Y}^{n}$ and $R_{z}^{n}$ be the truncation errors defined in (3.4) and (3.7), respectively. If $f_{1} \in C_{P}^{2,4}, h \in C_{b}^{2}$ and $\varphi \in C_{P}^{4}$,

$$
\begin{array}{ll}
\left|R_{y_{1}}^{n}\right| \leq C\left(1+\left|X^{n}\right|^{r}\right)(\Delta t)^{3}, & \left|R_{\Gamma_{1}}^{n}\right| \leq C\left(1+\left|X^{n}\right|^{r}\right)\left(\Delta t_{n}\right)^{3} \\
\left|R_{y_{2}}^{n}\right| \leq C\left(1+\left|X^{n}\right|^{r}\right)(\Delta t)^{3}, & \left|R_{\Gamma_{2}}^{n}\right| \leq C\left(1+\left|X^{n}\right|^{r}\right)\left(\Delta t_{n}\right)^{3} .
\end{array}
$$

Here $C$ is a positive constant depending only on $T$, and the upper bounds of $\varphi$ and $f$ and their derivatives.

Theorem 1 Suppose $f \in C_{P}^{2,4}, h \in C_{b}^{2}$ and $\varphi \in C_{P}^{4}$. Let $\left(X_{t}, Y_{t}, \Gamma_{t}\right)$ be the solution of the BSDE (1.1) and $\left(Y^{n}, Z^{n}\right)$ be the solution of scheme 1. Assume

$$
\begin{aligned}
& \mathbb{E}\left[\left|Y_{T}^{t_{N-1}, X^{N-1}}-Y^{N}\right|^{2}\right] \leq C\left(1+\left|X^{n}\right|^{r}\right)(\Delta t)^{4}, \\
& \mathbb{E}\left[\left|\Gamma_{T}^{t_{N-1}, X^{N-1}}-\Gamma^{N}\right|^{2}\right] \leq C\left(1+\left|X^{n}\right|^{r}\right)(\Delta t)^{2} .
\end{aligned}
$$

Then for sufficiently small time step $\Delta t$, we have

$$
\max _{0 \leq n \leq N} \mathbb{E}\left[\left|Y_{t_{n}}^{t_{n}, X^{n}}-Y^{n}\right|^{2}+\frac{1}{4}(\Delta t)^{2}\left|\Gamma_{t_{n}}^{t_{n}, X^{n}}-\Gamma^{n}\right|^{2}\right] \leq C\left(1+\left|X^{n}\right|^{r}\right)(\Delta t)^{4},
$$

where $C$ is a constant depending on $c_{0}, T$, and upper bounds of functions $h, \varphi$ and $f$ and their derivatives.

Proof. Let $e_{Y}^{n}=Y_{t_{n}}^{t_{n}, X^{n}}-Y^{n}$, and $e_{\Gamma}^{n}=\Gamma_{t_{n}}^{t_{n}, X^{n}}-\Gamma^{n}$. The Equations (3.10) and (3.6) give 


$$
\begin{aligned}
\frac{1}{2} \Delta t_{n} e_{\Gamma}^{n}= & -\frac{1}{2} \Delta t_{n} \mathbb{E}_{t_{n}}^{X^{n}}\left[e_{\Gamma}^{n+1}\right]+\mathbb{E}_{t_{n}}^{X^{n}}\left[e_{Y}^{n+1} \Delta \tilde{N}_{\rho, n}^{\mathrm{T}}\right] \\
& +\frac{1}{2} \Delta t_{n} \mathbb{E}_{t_{n}}^{X^{n}}\left[e_{f}^{n+1} \Delta \tilde{N}_{\rho, n}^{\mathrm{T}}\right]+\sum_{j=1}^{2} R_{\Gamma j}^{n} .
\end{aligned}
$$

Subtracting (3.9) from (3.3) gives

$$
\begin{aligned}
e_{Y}^{n}= & \mathbb{E}_{t_{n}}^{X^{n}}\left[e_{Y}^{n+1}\right]+\frac{1}{2} \Delta t_{n}\left\{f_{1}\left(t_{n}, Y_{t_{n}}^{t_{n}, X^{n}}\right)-f_{1}\left(t_{n}, Y^{n}\right)\right\}+\frac{1}{2} h\left(t_{n}\right) \Delta t_{n} e_{\Gamma}^{n} \\
& +\frac{1}{2} \Delta t_{n} \mathbb{E}_{t_{n}}^{X^{n}}\left[f_{1}\left(t_{n+1}, Y_{t_{n+1}}^{t_{n}, X^{n}}\right)-f_{1}\left(t_{n+1}, Y^{n+1}\right)\right] \\
& +\frac{1}{2} h\left(t_{n+1}\right) \Delta t_{n} \mathbb{E}_{t_{n}}^{X^{n}}\left[e_{\Gamma}^{n+1}\right]+\sum_{j=1}^{2} R_{y_{j}}^{n} .
\end{aligned}
$$

By the Hölder inequality (see [11] for details) we get

$$
\left|\mathbb{E}_{t_{n}}^{X^{n}}\left[e_{Y}^{n+1} \Delta \tilde{N}_{\rho, n}^{\mathrm{T}}\right]\right|^{2} \leq \Delta t_{n}\left(\mathbb{E}_{t_{n}}^{X^{n}}\left[\left|e_{Y}^{n+1}\right|^{2}\right]-\left|\mathbb{E}_{t_{n}}^{X^{n}}\left[e_{Y}^{n+1}\right]\right|^{2}\right)
$$

and

$$
\left|\mathbb{E}_{t_{n}}^{X^{n}}\left[h\left(t_{n+1}\right) e_{\Gamma}^{n+1} \Delta \tilde{N}_{\rho, n}^{\mathrm{T}}\right]\right|^{2} \leq C \Delta t_{n}\left(\mathbb{E}_{t_{n}}^{X^{n}}\left[\left|e_{\Gamma}^{n+1}\right|^{2}\right]-\left|\mathbb{E}_{t_{n}}^{X^{n}}\left[e_{\Gamma}^{n+1}\right]\right|^{2}\right)
$$

Then by the inequalities (4.6) and (4.7) we have

$$
\begin{aligned}
\left|\mathbb{E}_{t_{n}}^{X^{n}}\left[e_{Y}^{n+1} \Delta \tilde{N}_{\rho, n}^{\mathrm{T}}\right]\right|^{2} & =\left|\mathbb{E}_{t_{n}}^{X^{n}}\left[\left(e_{Y}^{n+1}-\mathbb{E}_{t_{n}}^{X^{n}}\left[e_{Y}^{n+1}\right]\right) \Delta \tilde{N}_{\rho, n}^{\mathrm{T}}\right]\right|^{2} \\
& \leq \mathbb{E}_{t_{n}}^{X^{n}}\left[\left(\Delta \tilde{N}_{\rho, n}^{\mathrm{T}}\right)^{2}\right] \mathbb{E}_{t_{n}}^{X^{n}}\left[\left(e_{Y}^{n+1}-\mathbb{E}_{t_{n}}^{X^{n}}\left[e_{Y}^{n+1}\right]\right)^{2}\right] \\
& \leq \int_{\mathcal{E}} \rho^{2}(e) \lambda(\mathrm{d} e) \Delta t_{n}\left(\mathbb{E}_{t_{n}}^{X^{n}}\left[\left|e_{Y}^{n+1}\right|^{2}\right]-\left|\mathbb{E}_{t_{n}}^{X^{n}}\left[e_{Y}^{n+1}\right]\right|^{2}\right) \\
& =\int_{\mathcal{E}} \rho^{2}(e) \lambda(\mathrm{d} e) \Delta t_{n} \operatorname{Var}^{n}\left(e_{Y}^{n+1}\right) .
\end{aligned}
$$

where $\operatorname{Var}^{n}(X)=\mathbb{E}_{t_{n}}^{X^{n}}\left[|X|^{2}\right]-\left|\mathbb{E}_{t_{n}}^{X^{n}}[X]\right|^{2}$. Inserting $\frac{1}{2} \Delta t e_{\Gamma}^{n} \quad$ which satisfies the Equation (4.4) into (4.5) leads to

$$
\begin{aligned}
e_{Y}^{n}= & \mathbb{E}_{t_{n}}^{X^{n}}\left[e_{Y}^{n+1}\right]+h\left(t_{n}\right) \mathbb{E}_{t_{n}}^{X^{n}}\left[e_{Y}^{n+1} \Delta \tilde{N}_{\rho, n}^{\mathrm{T}}\right]+\frac{1}{2} \Delta t_{n}\left\{f_{1}\left(t_{n}, Y_{t_{n}}^{t_{n}, X^{n}}\right)-f_{1}\left(t_{n}, Y^{n}\right)\right. \\
& \left.+\mathbb{E}_{t_{n}}^{X^{n}}\left[f_{1}\left(t_{n+1}, Y_{t_{n+1}}^{t_{n}, X^{n}}\right)-f_{1}\left(t_{n+1}, Y^{n+1}\right)\right]\right\}+\frac{1}{2} \Delta t_{n} \mathbb{E}_{t_{n}}^{X^{n}}\left[\left(h\left(t_{n+1}\right)-h\left(t_{n}\right)\right) e_{\Gamma}^{n+1}\right] \\
& +\frac{h\left(t_{n}\right)}{2} \Delta t_{n} \mathbb{E}_{t_{n}}^{X^{n}}\left[\left(f_{1}\left(t_{n+1}, Y_{t_{n+1}}^{t_{n}, X^{n}}\right)-f_{1}\left(t_{n+1}, Y^{n+1}\right)+h\left(t_{n+1}\right) e_{\Gamma}^{n+1}\right) \Delta \tilde{N}_{\rho, n}^{\mathrm{T}}\right] \\
& +h\left(t_{n}\right) \sum_{j=1}^{2} R_{\Gamma_{j}}^{n}+\sum_{j=1}^{2} R_{y_{j}}^{n} .
\end{aligned}
$$

By the Taylor expansion (see [11] for details) we get

$$
h\left(t_{n+1}-\right) h\left(t_{n}\right)=h_{t}^{\prime}\left(t_{n}+\alpha \Delta t\right) \Delta t,
$$

where $\alpha$ is a positive number which values in $[0,1]$. Thus, from the Hölder inequality, (4.9) and (4.10), we deduce 


$$
\begin{aligned}
\left|e_{Y}^{n}\right| \leq & \left|\mathbb{E}_{t_{n}}^{X^{n}}\left[e_{Y}^{n+1}\right]\right|+\left|h\left(t_{n}\right)\right|\left|\mathbb{E}_{t_{n}}^{X^{n}}\left[e_{Y}^{n+1} \Delta \tilde{N}_{\rho, n}^{\mathrm{T}}\right]\right| \\
& +\frac{1}{2} L \Delta t\left(\left|e_{Y}^{n}\right|+\mathbb{E}_{t_{n}}^{X^{n}}\left[\left|e_{Y}^{n+1}\right|\right]\right)+\frac{1}{2}\left|h_{t}^{\prime}\left(t_{n}+\alpha \Delta t\right)\right|(\Delta t)^{2} \mathbb{E}_{t_{n}}^{X^{n}}\left[\left|e_{\Gamma}^{n+1}\right|\right] \\
& +\frac{\left|h\left(t_{n}\right)\right|}{2} L \Delta t \mathbb{E}_{t_{n}}^{X^{n}}\left[\left|e_{Y}^{n+1}\right|^{2}\right]^{\frac{1}{2}} \mathbb{E}_{t_{n}}^{X^{n}}\left[\left|\Delta \tilde{N}_{\rho, n}^{\mathrm{T}}\right|^{2}\right]^{\frac{1}{2}} \\
& +\frac{\left|h\left(t_{n}\right)\right|\left|h\left(t_{n+1}\right)\right|}{2} \Delta t\left|\mathbb{E}_{t_{n}}^{X^{n}}\left[e_{\Gamma}^{n+1} \Delta \tilde{N}_{\rho, n}^{\mathrm{T}}\right]\right|+\left|h\left(t_{n}\right)\right| \sum_{j=1}^{2}\left|R_{\Gamma_{j}}^{n}\right|+\sum_{j=1}^{2}\left|R_{y_{j}}^{n}\right|,
\end{aligned}
$$

where $L$ is the Lipschitz constant of $f_{1}(t, y)$ with respect to $y$. Then by the inequalities (4.11) and $(a+b)^{2} \leq\left(1+\gamma_{1} \Delta t\right) a^{2}+\left(1+\frac{1}{\gamma_{1} \Delta t}\right) b^{2}$, we have

$$
\begin{aligned}
& \left|e_{Y}^{n}\right|^{2} \leq\left(1+\gamma_{1} \Delta t\right)\left|\mathbb{E}_{t_{n}}^{X^{n}}\left[e_{Y}^{n+1}\right]\right|^{2}+\left(1+\frac{1}{\gamma_{1} \Delta t}\right) C\left(\left|\mathbb{E}_{t_{n}}^{X^{n}}\left[e_{Y}^{n+1} \Delta \tilde{N}_{\rho, n}^{\mathrm{T}}\right]\right|\right. \\
& +\Delta t\left(\left|e_{Y}^{n}\right|+\mathbb{E}_{t_{n}}^{X^{n}}\left[\left|e_{Y}^{n+1}\right|\right]\right)+(\Delta t)^{2} \mathbb{E}_{t_{n}}^{X^{n}}\left[\left|e_{\Gamma}^{n+1}\right|\right]+(\Delta t)^{\frac{3}{2}} \mathbb{E}_{t_{n}}^{X^{n}}\left[\left|e_{Y}^{n+1}\right|^{2}\right]^{\frac{1}{2}} \\
& \left.+\Delta t\left|\mathbb{E}_{t_{n}}^{X^{n}}\left[e_{\Gamma}^{n+1} \Delta \tilde{N}_{\rho, n}^{\mathrm{T}}\right]\right|+\sum_{j=1}^{2}\left|R_{\Gamma_{j}}^{n}\right|+\sum_{j=1}^{2}\left|R_{y_{j}}^{n}\right|\right)^{2} \\
& \leq\left(1+\gamma_{1} \Delta t\right)\left|\mathbb{E}_{t_{n}}^{X^{n}}\left[e_{Y}^{n+1}\right]\right|^{2}+C \frac{1+\gamma_{1} \Delta t}{\gamma_{1}} \mid \Delta t\left(\left|e_{Y}^{n}\right|^{2}+\mathbb{E}_{t_{n}}^{X^{n}}\left[\left|e_{Y}^{n+1}\right|^{2}\right]\right) \\
& \left.+\operatorname{Var}^{n}\left[e_{Y}^{n+1}\right]+(\Delta t)^{3} \mathbb{E}_{t_{n}}^{X^{n}}\left[\left|e_{\Gamma}^{n+1}\right|^{2}\right]+(\Delta t)^{2} \operatorname{Var}^{n}\left[e_{\Gamma}^{n+1}\right]+\sum_{j=1}^{2} \frac{\left|R_{y_{j}}^{n}\right|^{2}+\left|R_{\Gamma_{j}}^{n}\right|^{2}}{\Delta t}\right) .
\end{aligned}
$$

The Equation (4.4), the inequalities (4.6), (4.12) and

$$
\begin{aligned}
& (a+b)^{2} \leq\left(1+\gamma_{2} \Delta t\right) a^{2}+\left(1+\frac{1}{\gamma_{2} \Delta t}\right) b^{2} \text { yield } \\
& \frac{1}{4}\left(\Delta t_{n}\right)^{2}\left(e_{\Gamma}^{n}\right)^{2} \\
& \leq \frac{1}{4}\left(\Delta t_{n}\right)^{2}\left(1+\gamma_{2} \Delta t\right)\left(\mathbb{E}_{t_{n}}^{X^{n}}\left[e_{\Gamma}^{n+1}\right]\right)^{2} \\
& +\left(1+\frac{1}{\gamma_{2} \Delta t}\right)\left(\mathbb{E}_{t_{n}}^{X^{n}}\left[e_{Y}^{n+1} \Delta \tilde{N}_{\rho, n}^{\mathrm{T}}\right]+\frac{1}{2} \Delta t \mathbb{E}_{t_{n}}^{X^{n}}\left[e_{f}^{n+1} \Delta W_{t_{n+1}}^{\mathrm{T}}\right]+\sum_{j=1}^{2} R_{\Gamma_{j}}^{n}\right)^{2} \\
& \leq \frac{1}{4}\left(\Delta t_{n}\right)^{2}\left(1+\gamma_{2} \Delta t\right)\left(\mathbb{E}_{t_{n}}^{X^{n}}\left[e_{\Gamma}^{n+1}\right]\right)^{2}+C \frac{1+\gamma_{2} \Delta t}{\gamma_{2}}\left(\mathbb{E}_{t_{n}}^{X^{n}}\left[\left|e_{Y}^{n+1}\right|^{2}\right]-\left|\mathbb{E}_{t_{n}}^{X^{n}}\left[e_{Y}^{n+1}\right]\right|^{2}\right. \\
& \left.\quad+\left(\Delta t_{n}\right)^{2}\left(\mathbb{E}_{t_{n}}^{X^{n}}\left[\left|e_{\Gamma}^{n+1}\right|^{2}\right]-\left|\mathbb{E}_{t_{n}}^{X^{n}}\left[e_{\Gamma}^{n+1}\right]\right|^{2}\right)+\Delta t \mathbb{E}_{t_{n}}^{X^{n}}\left[\left|e_{Y}^{n+1}\right|^{2}\right]+\sum_{j=1}^{2} \frac{\left.R_{\Gamma_{j}}^{n}\right|^{2}}{\Delta}\right) .
\end{aligned}
$$

Multiplying both sides of (4.13) by $\frac{(\Delta t)^{2}}{\left(\Delta t_{n}\right)^{2}}$ implies 


$$
\begin{aligned}
\frac{1}{4}(\Delta t)^{2}\left(e_{\Gamma}^{n}\right)^{2} \leq & \frac{1}{4}(\Delta t)^{2}\left(1+\gamma_{2} \Delta t\right)\left(\mathbb{E}_{t_{n}}^{X^{n}}\left[e_{\Gamma}^{n+1}\right]\right)^{2}+C \frac{1+\gamma_{2} \Delta t}{\gamma_{2}}\left(\operatorname{Var}^{n}\left[e_{Y}^{n+1}\right]\right. \\
& \left.+(\Delta t)^{2} \operatorname{Var}^{n}\left[e_{\Gamma}^{n+1}\right]+\Delta t \mathbb{E}_{t_{n}}^{X^{n}}\left[\left|e_{Y}^{n+1}\right|^{2}\right]+\sum_{j=1}^{2} \frac{\left|R_{\Gamma_{j}}^{n}\right|^{2}}{\Delta t}\right)
\end{aligned}
$$

Now by the inequalities (4.12) and (4.14) we get

$$
\begin{aligned}
& \left|e_{Y}^{n}\right|^{2}+\frac{1}{4}(\Delta t)^{2}\left(e_{\Gamma}^{n}\right)^{2} \\
& \leq\left(1+\gamma_{1} \Delta t\right)\left|\mathbb{E}_{t_{n}}^{X^{n}}\left[e_{Y}^{n+1}\right]\right|^{2}+\frac{1}{4}(\Delta t)^{2}\left(1+\gamma_{2} \Delta t\right)\left|\mathbb{E}_{t_{n}}^{X^{n}}\left[e_{\Gamma}^{n+1}\right]\right|^{2} \\
& +C \frac{1+\gamma_{1} \Delta t}{\gamma_{1}}\left(\Delta t\left(\left|e_{Y}^{n}\right|^{2}+\mathbb{E}_{t_{n}}^{X^{n}}\left[\left|e_{Y}^{n+1}\right|^{2}\right]\right)+(\Delta t)^{3} \mathbb{E}_{t_{n}}^{X^{n}}\left[\left|e_{\Gamma}^{n+1}\right|^{2}\right]\right. \\
& +(\Delta t)^{2} \operatorname{Var}^{n}\left[e_{\Gamma}^{n+1}\right]+\sum_{j=1}^{2} \frac{\left|R_{y_{j}}^{n}\right|^{2}}{\Delta t}+C \frac{1+\gamma_{2} \Delta t}{\gamma_{2}} \\
& +\left(\operatorname{Var}^{n}\left[e_{Y}^{n+1}\right]+(\Delta t)^{2} \operatorname{Var}^{n}\left[e_{\Gamma}^{n+1}\right]+\Delta t \mathbb{E}_{t_{n}}^{X^{n}}\left[\left|e_{Y}^{n+1}\right|^{2}\right]+\sum_{j=1}^{2} \frac{\left|R_{\Gamma_{j}}^{n}\right|^{2}}{\Delta t}\right) .
\end{aligned}
$$

Choosing $\gamma_{1}=\gamma_{2}=8 C$ such that $\frac{C}{\gamma_{1}}=\frac{1}{8}$ and $\frac{C}{\gamma_{2}}=\frac{1}{8}$ in (4.15), and using the estimate

$$
\begin{aligned}
& (1+8 C \Delta t)\left(\left|\mathbb{E}_{t_{n}}^{X^{n}}\left[e_{Y}^{n+1}\right]\right|^{2}+\frac{1}{8}\left(\mathbb{E}_{t_{n}}^{X^{n}}\left[\left|e_{Y}^{n+1}\right|^{2}\right]-\left|\mathbb{E}_{t_{n}}^{X^{n}}\left[e_{Y}^{n+1}\right]\right|^{2}\right)\right) \\
& \leq(1+8 C \Delta t) \mathbb{E}_{t_{n}}^{X^{n}}\left[\left|e_{Y}^{n+1}\right|^{2}\right], \\
& \frac{1+8 C \Delta t}{8}(\Delta t)^{2}\left(2\left|\mathbb{E}_{t_{n}}^{X^{n}}\left[e_{\Gamma}^{n+1}\right]\right|^{2}+\mathbb{E}_{t_{n}}^{X^{n}}\left[\left|e_{\Gamma}^{n+1}\right|^{2}\right]-\left|\mathbb{E}_{t_{n}}^{X^{n}}\left[e_{\Gamma}^{n+1}\right]\right|^{2}\right) \\
& \leq \frac{1}{4}(\Delta t)^{2}(1+8 C \Delta t) \mathbb{E}_{t_{n}}^{X^{n}}\left[\left|e_{\Gamma}^{n+1}\right|^{2}\right],
\end{aligned}
$$

we deduce

$$
\begin{aligned}
& \left|e_{Y}^{n}\right|^{2}+\frac{1}{4}(\Delta t)^{2}\left(e_{\Gamma}^{n}\right)^{2} \\
& \leq(1+8 C \Delta t) \mathbb{E}_{t_{n}}^{X^{n}}\left[\left|e_{Y}^{n+1}\right|^{2}\right]+\frac{1}{4}(\Delta t)^{2}(1+8 C \Delta t) \mathbb{E}_{t_{n}}^{X^{n}}\left[\left|e_{\Gamma}^{n+1}\right|^{2}\right] \\
& \quad+\frac{1+8 C \Delta t}{8}\left(\Delta t\left(\left|e_{Y}^{n}\right|^{2}+\mathbb{E}_{t_{n}}^{X^{n}}\left[\left|e_{Y}^{n+1}\right|^{2}\right]\right)+(\Delta t)^{3} \mathbb{E}_{t_{n}}^{X^{n}}\left[\left|e_{\Gamma}^{n+1}\right|^{2}\right]\right. \\
& \left.\quad+\Delta t \mathbb{E}_{t_{n}}^{X^{n}}\left[\left|e_{Y}^{n+1}\right|^{2}\right]\right)+C \sum_{j=1}^{2} \frac{\left|R_{\Gamma_{j}}^{n}\right|^{2}+\left|R_{y_{j}}^{n}\right|^{2}}{\Delta t} \\
& \leq(1+C \Delta t) \mathbb{E}_{t_{n}}^{X^{n}}\left[\left|e_{Y}^{n+1}\right|^{2}+\frac{1}{4}(\Delta t)^{2}\left|e_{\Gamma}^{n+1}\right|^{2}\right]+C \Delta t\left|e_{Y}^{n}\right|^{2}+C \sum_{j=1}^{2} \frac{\left|R_{\Gamma_{j}}^{n}\right|^{2}+\left|R_{y_{j}}^{n}\right|^{2}}{\Delta t} .
\end{aligned}
$$

By Lemma 1, we have $\left|R_{y_{j}}^{n}\right| \leq C(\Delta t)^{3} \leq C(\Delta t)^{3}$ and $\left|R_{\Gamma_{j}}^{n}\right| \leq C(\Delta t)^{3} \leq C(\Delta t)^{3}$ 
for $j=1,2$. Taking mathematical expectation on both sides of (4.16), then for sufficiently small $\Delta t$, we have

$$
\begin{aligned}
& \mathbb{E}\left[\left|e_{Y}^{n}\right|^{2}+\frac{1}{4}(\Delta t)^{2}\left(e_{\Gamma}^{n}\right)^{2}\right] \\
& \leq \frac{1+C \Delta t}{1-C \Delta t} \mathbb{E}\left[\left|e_{Y}^{n+1}\right|^{2}+\frac{1}{4}(\Delta t)^{2}\left|e_{\Gamma}^{n+1}\right|^{2}\right]+\frac{C(\Delta t)^{5}}{1-C \Delta t} \\
& \leq\left(\frac{1+C \Delta t}{1-C \Delta t}\right)^{N-n} \mathbb{E}\left[\left|e_{Y}^{N}\right|^{2}+\frac{1}{4}(\Delta t)^{2}\left|e_{\Gamma}^{N}\right|^{2}\right]+\frac{C(\Delta t)^{5}}{1-C \Delta t} \sum_{i=0}^{N-n-1}\left(\frac{1+C \Delta t}{1-C \Delta t}\right)^{i}
\end{aligned}
$$

for $n=N-1, \cdots, 0$. The terminal conditions $\mathbb{E}\left[\left|e_{Y}^{N}\right|^{2}\right] \leq C(\Delta t)^{4}$ and $\mathbb{E}\left[\left|e_{\Gamma}^{N}\right|^{2}\right] \leq C(\Delta t)^{2}$, the time step constraint (4.1), and the inequality

$$
\frac{C(\Delta t)^{5}}{1-C \Delta t} \sum_{i=0}^{N-n-1}\left(\frac{1+C \Delta t}{1-C \Delta t}\right)^{i} \leq \frac{(\Delta t)^{4}}{2}\left(e^{2 c_{0} C T}-1\right)
$$

lead to

$$
\mathbb{E}\left[\left|e_{Y}^{n}\right|^{2}+\frac{1}{4}(\Delta t)^{2}\left|e_{\Gamma}^{n}\right|^{2}\right] \leq C(\Delta t)^{4}
$$

for $n=N, N-1, \cdots, 0$. The proof is completed.

\section{Conclusion}

In this paper, we study the error estimate of the Crank-Nicolson scheme proposed in [10] for solving a kind of decoupled FBSDEs. Under weaker conditions than that in [9], we rigorously prove the second order convergence rate of the Crank-Nicolson scheme.

\section{References}

[1] Pardoux, E. and Peng, S. (1990) Adapted Solution of a Backward Stochastic Differntial Equation. Systems \& Control Letters, 14, 55-61.

https://doi.org/10.1016/0167-6911(90)90082-6

[2] Peng, S. (1990) Probabilistic Interpretation for Systems of Quasilinear Parabolic Partial Differential Equations. Stochastics and Stochastics Reports, 37, 61-74.

[3] Ma, J., Protter, P. and Yong, J. (1994) Solving Forward-Backward Stochastic Differential Equations Explicitly-A Four Step Scheme. Probability Theory and Related Fields, 98, 339-359. https://doi.org/10.1007/BF01192258

[4] Zhang, J. (2004) A Numerical Scheme for BSDEs. Annals of Applied Probability, 14, 459-488. https://doi.org/10.1214/aoap/1075828058

[5] Zhao, W., Chen, L. and Peng, S. (2006) A New Kind of Accurate Numerical Method for Backward Stochastic Differential Equations. SIAM: SIAM Journal on Scientific Computing, 28, 1563-1581. https://doi.org/10.1137/05063341X

[6] Wang, J.L., Luo, C.X. and Zhao, W.D. (2009) Crank-Nicolson Scheme and Its Error Estimates for Backward Stochastic Differential Equations. Acta Mathematicae Applicatae Sinica (English Series).

[7] Zhao, W., Wang, J. and Peng, S. (2009) Error Estimates of the $\theta$-Scheme for Back- 
ward Stochastic Differential Equations. Discrete and Continuous Dynamical Systems-Series B, 12, 905-924. https://doi.org/10.3934/dcdsb.2009.12.905

[8] Li, Y. and Zhao, W. (2010) $L^{p}$-Error Estimates for Numerical Schemes for Solving Certain Kinds of Backward Stochastic Differential Equations. Statistics and Probability Letters, 80, 1612-1617.

[9] Li, Y., Yang, J. and Zhao, W. (2017) Convergence Error Estimates of the CrankNicolson Scheme for Solving Decoupled FBSDEs. Science China Mathematics, 60, 923-948. https://doi.org/10.1007/s11425-016-0178-8

[10] Zhao, W., Li, Y. and Fu, Y. (2014) Second-Order Schemes for Solving Decoupled Forward Backward Stochastic Differential Equations. Science China Mathematics, 57, 665-686. https://doi.org/10.1007/s11425-013-4764-0

[11] Revuz, D. and Yor, M. Continuous Martingales and Brownian Motion. 